\title{
Presentación de un caso de arteritis de Takayasu en un adolescente
}

\author{
Takayasu arteritis in an adolescent, case report
}

\author{
Franklin Aranda-Paniora ${ }^{1,2}$, Sandra Schult Montoya ${ }^{1, a}$, Patricia Ponce Vilca ${ }^{1, a}$ \\ 'Médico Pediatra, Asistente del Servicio de Medicina "D”, Instituto Nacional de Salud del Niño, Lima Perú. \\ ${ }^{2}$ Docente de Pediatría, Facultad de Medicina, Universidad Nacional Mayor de San Marcos, Lima, Perú.
}

\begin{abstract}
Resumen
La arteritis de Takayasu es una entidad rara en pediatría y su presentación clínica plantea diversos diagnósticos diferenciales. Se publica el caso de un adolescente de 14 años que ingresó a un Hospital de Lima con convulsiones tónico clónicas generalizadas e hipertensión arterial de grado 2. Posteriormente fue derivado para estudio al Instituto de Salud del Niño de Lima, Perú. Se revisa los criterios diagnósticos, su evolución y tratamiento.
\end{abstract}

Palabras clave. Arteritis de Takayasu; Hipertensión Arterial; Pediatría.

Abstract

Takayasu arteritis is a rare entity in pediatrics and clinical presentation raises several differential diagnoses. We report the case of a 14-year-old boy with generalized tonic clonic seizures and stage 2 hypertension referred for study to the Instituto Nacional de Salud del Niño in Lima, Peru. Diagnostic criteria, evolution and treatment are reviewed.

Keywords. Takayasu's Arteritis; Hypertension; Pediatrics.

An Fac med. 2016;77(2):167-70 / http://dx.doi.org/10.15381/anales.v77i2.11822

\section{INTRODUCCIÓN}

La arteritis de Takayasu es una vasculitis de grandes vasos de origen desconocido mediada por células $\mathrm{T}$ sin anticuerpos órgano-específicos, aunque se describe de forma ocasional anticuerpos antiaorta, antiendotelio y antianexina $\mathrm{V}^{(1)}$. Al ser una enfermedad inflamatoria crónica que afecta la aorta y sus ramas principales, puede cursar con engrosamiento de pared vascular, fibrosis, estenosis y formación de trombos. La clínica puede ser el reflejo de la isquemia final del órgano afectado. Este proceso inflamatorio puede conducir a la destrucción de la capa media arterial y formación de aneurismas ${ }^{(2)}$.

Aunque se reporta que es una enfermedad más frecuente en mujeres entre la segunda y tercera década de la vida (3), ahora se reconoce su distribución global y en ambos sexos, aunque la manifestación de la enfermedad varía entre las poblaciones.
En 1908, Takayasu, profesor de oftalmología de la Universidad de Kanazawa, Japón, presentó en la $12^{\circ}$ Congreso Anual de la Sociedad de Oftalmología de Japón el caso de una mujer de 21 años con anastomosis arteriovenosa alrededor de la papila óptica ${ }^{(4)}$. Pero fue Yamamoto quien realizó una primera descripción en 1830 en su libro Kitsuo-Idan, donde describe a un paciente de 45 años con fiebre persistente, pérdida de peso y disnea sin pulso carotídeo y de miembros superiores ${ }^{(5)}$.

La arteritis de Takayasu ha sido encontrada en lactantes de seis meses hasta adultos de todas las edades y es una de las mayores causas de hipertensión renovascular en pediatría ${ }^{(6)}$ y la tercera vasculitis más común en pediatría después de la púrpura de Henoch Schonlein y la enfermedad de Kawasaki (7).

Una asociación entre arteritis de Takayasu e infección por Mycobacterium tuberculosis se ha observado en países donde la tuberculosis es en- démica, aunque no se ha probado su relación causal. En una serie de casos estudiados entre los 60s y 70s en Japón, Corea, India, México y Sudáfrica, se halló PPD positivo en $81 \%$ a $100 \%$ de los pacientes con Takayasu y en $21,8 \%$ a $50 \%$ se demostró tuberculosis activa. En comparación, solo 0,028\% a 0,309\% de la población general de estos países tuvo tuberculosis activa en esos años ${ }^{(8)}$.

La edad promedio de presentación es 11,4 años; se diagnostica antes de los 19 años en $20 \%$ de los casos y antes de los 10 años en $2 \%$ de los casos. Se tiene un retraso del diagnóstico de 19 meses ${ }^{(9)}$.

El cuadro clínico es inespecífico en la edad pediátrica, consistiendo en fiebre, mal estado general, anorexia, mialgias, artralgias, dolor abdominal, hipertensión arterial sistémica, retinopatía hipertensiva, insuficiencia cardiaca, cefalea y crisis convulsivas ${ }^{(9)}$.

El Colegio Americano de Reumatología ha establecido criterios diagnósti- 
cos de la arteritis de Takayasu, siendo 3 de 6 criterios necesarios. La presencia de tres o más criterios tiene una sensibilidad de $90,5 \%$ y especificidad de $97,8 \%{ }^{(10)}$ :

- Inicio de enfermedad de 40 años o menos.

- Claudicación de extremidades.

- Disminución del pulso de una o ambas arterias braquiales.

- Diferencia de al menos $10 \mathrm{mmHg}$ en la presión sistólica entre brazos.

- Soplo sobre una o ambas arterias subclavias o la aorta abdominal

- Estrechamiento arteriográfico u oclusión de toda la aorta, sus ramas proximales o grandes arterias de las extremidades superiores o inferiores que no son debidas a arterioesclerosis, displasia fibromuscular u otras.

Otros criterios diagnósticos fueron definidos por Ishikawa, en 1988 (2 criterios mayores o 1 criterio mayor y 2 menores, o con 4 criterios menores):

Mayores: edad inferior a 40 años (obligatorio), afectación de la arteria subclavia. Menores: aumento de la velocidad de sedimentación, aumento de la presión arterial, dolor en el territorio de la arteria carótida, lesiones arteriales ubicadas en aorta, tronco braquiocefálico, arteria carótida primitiva, arteria pulmonar ${ }^{(11)}$.

La arteritis de Takayasu puede ser clasificada en seis tipos, basado en criterios hagiográficos ${ }^{(12)}$.

- Tipo I - Ramas del arco aórtico

- Tipo Ila - Aorta ascendente, arco aórtico y sus ramas

- Tipo IIb - Región tipo IIa más aorta descendente torácica

- Tipo III - Aorta descendente torácica, aorta abdominal, arterias renales o una combinación

- Tipo IV - Aorta abdominal, arteria renal o ambas

- Tipo V - Toda la aorta y sus ramas.

\section{CASO CLÍNICO}

Paciente varón de 14 años de edad sin antecedentes relevantes, salvo episodios aislados y autolimitados de cefalea e inyección conjuntival seis meses previos. Presentó bruscamente cefalea, hiporexia y posteriormente convulsión tónico clónica generalizada, por lo que es conducido a un hospital de II nivel, donde tuvo además tres convulsiones en un lapso de tres horas, por lo que fue derivado a la unidad de cuidados intensivos. Se comprobó hipertensión arterial, iniciándose tratamiento antihipertensivo, con estabilización de la presión arterial en 140/100 mmHg. En los 10 días que permaneció hospitalizado, con diagnóstico de estatus convulsivo y probable lupus eritematoso sistémico, recibió pulso de metilprednisolona por tres días. Los estudios realizados en ese centro asistencial determinaron hipoplasia renal bilateral con signos de hipertensión renovascular por ecoDoppler arterial, tomografía espiral multicorte abdominal sin alteraciones, anticuerpos antinucleares y anticuerpos anticitoplasma del neutrófilo negativos. Fue dado de alta con fenitoína 100 mg cada 8 horas, alfametildopa $750 \mathrm{mg}$ cada 8 horas y nifedipino $30 \mathrm{mg}$ cada 12 horas. Tres días después del alta, la madre comprobó presión arterial de 190/110 mmHg, acudiendo el paciente a la emergencia del Instituto Nacional de Salud del Niño (INSN) donde se hospitalizó para estudio, por persistir la hipertensión a pesar de tratamiento con $30 \mathrm{mg}$ de nifedipino.

Ingresó dos días después al Servicio de Medicina D, con $60 \mathrm{~kg}$ de peso, $175 \mathrm{~cm}$ de talla, frecuencia cardiaca y respiratoria de 92 y 20 por minuto, respectivamente, y presión arterial 130/90 mmHg. El examen clínico no mostró otros hallazgos, salvo la disminución de ambos pulsos pedios. Los exámenes de laboratorio son presentados en la tabla 1.

Se inició tratamiento con alfametildopa $500 \mathrm{mg}$ cada 8 horas y nifedipino 30 mg cada 8 horas, con persistencia de presiones por encima de percentil 95. Dos semanas después se agregó carvedilol 12,5 mg cada 24 horas, estabilizándose la presión en $140 / 70 \mathrm{mmHg}$, con crisis hipertensivas durante las madrugadas, las que cedían con nifedipino $10 \mathrm{mg}$ sublingual. Debido a las variaciones frecuentes de la presión arterial

Tabla 1. Resultados hematológicos, bioquímicos e inmunológicos.

\begin{tabular}{|c|c|c|c|c|c|}
\hline \multicolumn{2}{|c|}{ Hematológicos } & \multicolumn{2}{|c|}{ Bioquímicos } & \multicolumn{2}{|c|}{ Inmunológicos } \\
\hline Hemoglobina & 12,7 & Glucosa & 90 & C3 & 228 \\
\hline Hematocrito & 38 & Urea & 103 & ASO & 21 \\
\hline Leucocitos & 10300 & Creatinina & 1,36 & TSH & 5,42 \\
\hline Abastonados & $0 \%$ & Sodio & 131 & T4 libre & 17,2 \\
\hline Plaquetas & & Potasio & 4,3 & Anti TPO & $<10$ \\
\hline TTPa razón & 1,54 & Cloro & 91 & Anti TG & $<20$ \\
\hline TP razón & 1,05 & Calcio & 11,1 & HIV & No reactivo \\
\hline Grupo y factor & $0+$ & Fosforo & 5,6 & $\mathrm{AgHBs}$ & No reactivo \\
\hline & & Fosfat. alcalina & 578 & & \\
\hline \multicolumn{2}{|c|}{ Ecocardio } & TGP & 149 & & \\
\hline \multirow{3}{*}{$\begin{array}{l}\text { Leve hipertrofia } \\
\text { concéntrica } \\
\text { de ventrículo } \\
\text { izquierdo }\end{array}$} & & TGO & 116 & & \\
\hline & & PCR & & & \\
\hline & & $\mathrm{DHL}$ & 401 & & \\
\hline \multicolumn{6}{|c|}{ Orina } \\
\hline Examen de orina & $\mathrm{Hb}$ trazas & $\begin{array}{c}\text { Proteinuria } 24 \\
\text { horas }\end{array}$ & Negativo & $\begin{array}{c}\text { Metanefrinas y } \\
\text { Normetanefrinas en } \\
\text { orina de } 24 \text { horas }\end{array}$ & Negativos \\
\hline
\end{tabular}




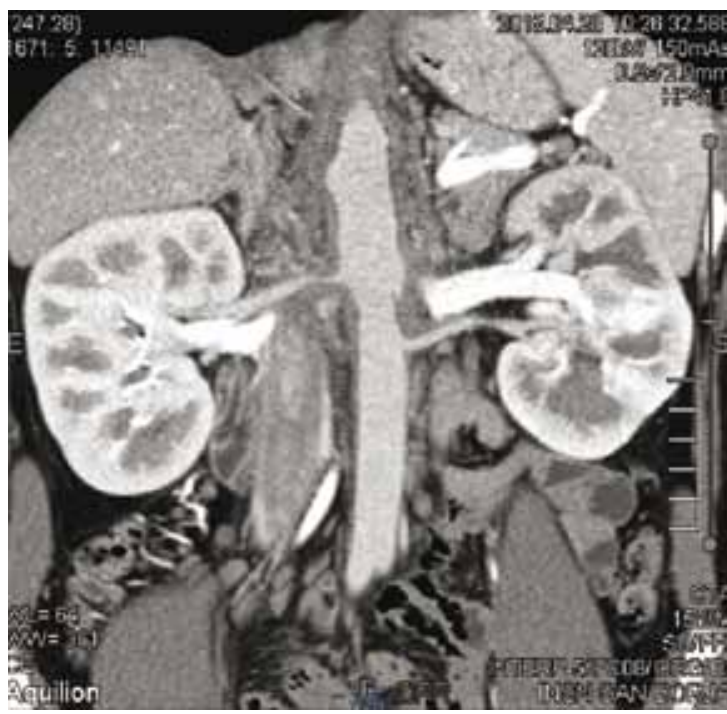

Figura 1. Engrosamiento de la pared de ambas arterias renales con predominio derecho, ocasionando estenosis significativa de su luz, más de 70\%, que llega a medir hasta 1,5 mm de calibre.

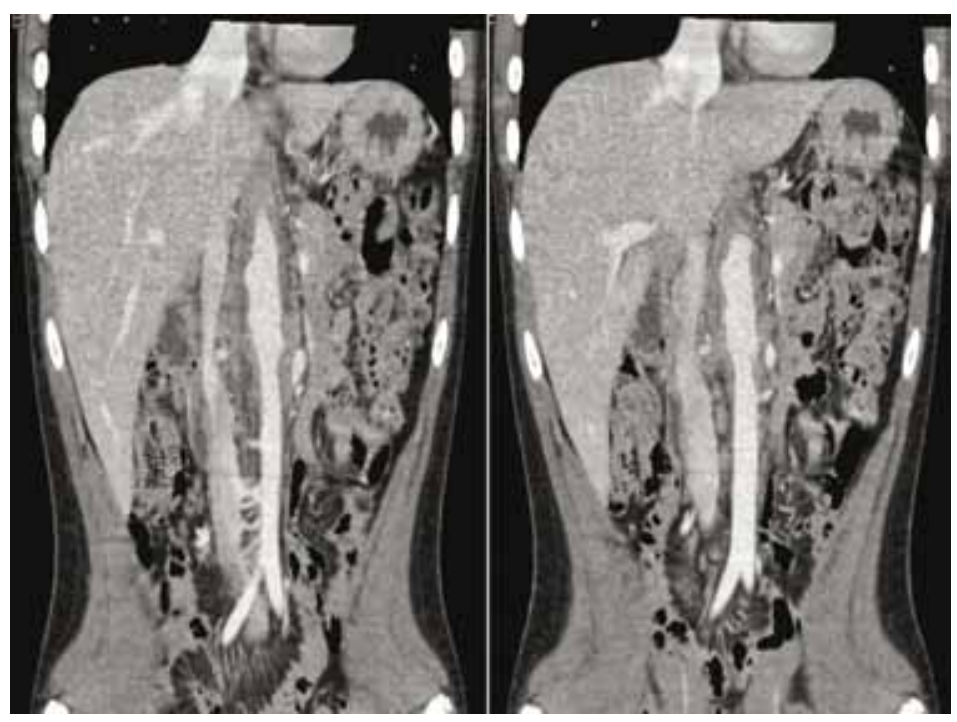

Figura 2. Engrosamiento difuso de la pared arterial de la aorta a nivel diafragmático y abdominal, así como del origen de sus ramas intraabdominales, con estenosis de la arteria gástrica izquierda. durante la evolución, el esquema antihipertensivo fue cambiado hasta estabilizarlo con alfametildopa $500 \mathrm{mg}$ cada 8 horas, amlodipino $5 \mathrm{mg}$ cada 24 horas, carvedilol $12,5 \mathrm{mg}$ cada 12 horas y nifedipino $10 \mathrm{mg}$ sublingual condicional a presiones superiores al percentil 95.

Se obtuvo angiorresonancia abdominal y cerebral seis días después del ingreso al servicio. Los hallazgos a nivel de aorta abdominal y arteria renal se presentan en las figuras 1 y 2 . No se encontraron alteraciones en arterias cerebrales.

Al cumplir los criterios de Ishikawa para arteritis de Takayasu, se procedió a manejo multidisciplinario. Se inició tratamiento con metotrexato $20 \mathrm{mg}$ semanal y prednisona $25 \mathrm{mg} /$ día, a los 15 días de ingreso al servicio. Debido a la relación de tuberculosis y arteritis de Takayasu ${ }^{(13)}$, se solicitó interferón gamma (Quantiferon TB gold), resultando positivo. Ante ausencia de otros hallazgos en la tomografía espiral multicorte de tórax, se planteó el diagnostico de tuberculosis latente y se inició tratamiento con isoniacida $300 \mathrm{mg}$, a las tres semanas después de su ingreso al servicio.
A las cinco semanas de su ingreso, se realizó arteriografía renal, que se observa en las figuras 3 y 4.

A la duodécima semana del ingreso, se realizó angioplastia con stent de arteria renal derecha $(5 \times 19 \mathrm{~mm})$ e izquierda $(4 \times 16 \mathrm{~mm})$, exitosamente, y se indicó iniciar tratamiento con clopidogrel $75 \mathrm{mg} / \mathrm{dí}$. Se fue retirando progresivamente el carvedilol y la metildopa hasta suspenderlo definitivamente al quinto día post colocación del stent. Fue dado de alta una semana después, con hemoglobina en 13,6 gr/ $\mathrm{dL}$, sin alteraciones en el hemograma ni en el perfil de coagulación y con niveles normales de transaminasa, urea y creatinina, manteniendo el tratamiento con amlodipino, prednisona, isoniacida, metotrexato semanal y pulsos mensuales de ciclofosfamida.

El paciente reinició sus rutinas habituales y la actividad escolar sin alteraciones clínicas ni de la presión arterial. A la cuarta semana del alta, los controles hematológicos mostraron un hematocrito en 41\%, 9670 leucocitos/ $\mathrm{mL}, 247000$ plaquetas $/ \mathrm{mL}$, velocidad de sedimentación $30 \mathrm{~mm} /$ hora, urea 27 $\mathrm{mg} / \mathrm{dL}$, creatinina $0,63 \mathrm{mg} / \mathrm{dL}$, tiempo de protrombina y tiempo de trombo- plastina parcial activada normales. Se realizó una ecografía Doppler de carótidas y subclavias a las 8 semanas del alta, sin alteraciones.

Hasta la vigésima cuarta semana del alta, el paciente permanecía asintomático, con reactantes de fase aguda normales y la presión arterial controlada, manteniendo su tratamiento con amlodipino, metotrexato semanal, 10 mg prednisona diario, clopidogrel, habiendo cumplido seis pulsos de ciclofosfamida y seis meses de tratamiento con isoniacida.

\section{DISCUSIÓN}

La arteritis de Takayasu es una vasculitis de grandes vasos de presentación rara, por lo que su enfoque y manejo en la edad pediátrica presenta dificultades. Si bien el cuadro clínico es inespecífico, es una de las principales causas de hipertensión renovascular; es un diagnóstico diferencial en la edad pediátrica.

$\mathrm{Su}$ asociación con tuberculosis ha sido comunicada en diversas publicaciones, aunque no su causalidad; es necesario entonces su descarte en un medio con alta prevalencia como el nuestro. 


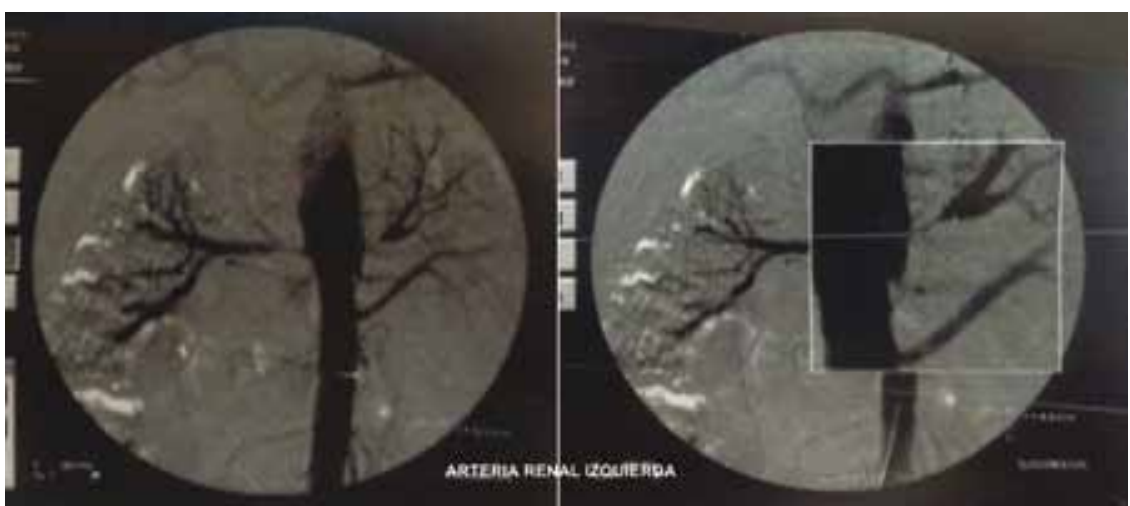

Figura 3. Doble arteria renal izquierda con marcada estenosis (77\%) del ramo superior, desde su ostium aórtico.

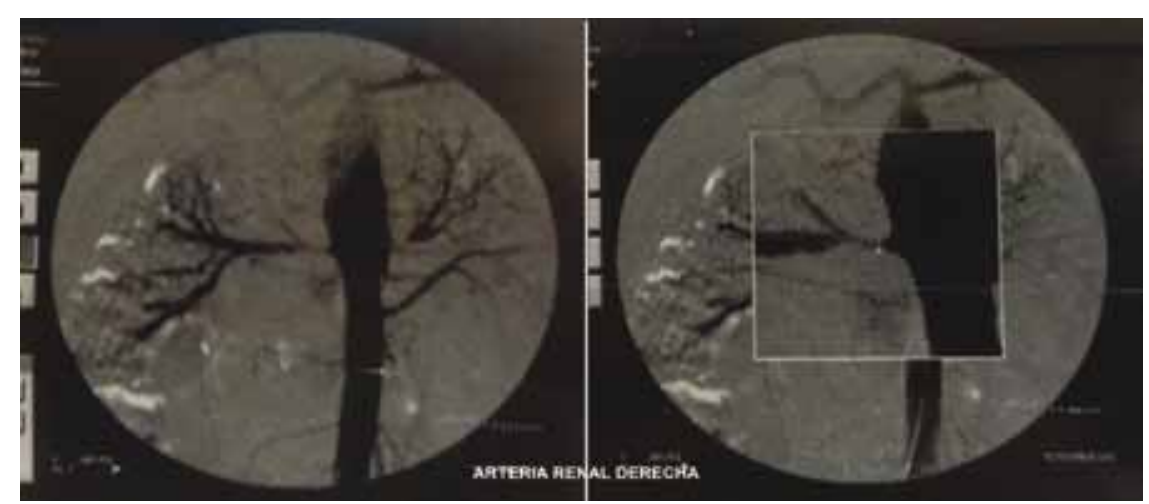

Figura 4. Estenosis (30\%) de la arteria renal derecha.

La corticoterapia y la inmunosupresión es el tratamiento actualmente indicado. Los corticoides han demostrado disminuir hasta en $50 \%$ la progresión de las lesiones y la terapia inmunosupresora evita la re-estenosis ${ }^{(14)}$. $\mathrm{Al}$ ser irreversible la estenosis, la angioplastia precoz es necesaria para el manejo de la hipertensión renovascular y prevención de complicaciones ${ }^{(15)}$.

El manejo principal de la hipertensión se centra en la realización de la angioplastia, que en el caso de nuestro paciente fue fundamental para el control de la hipertensión.

No existen publicaciones acerca del enfoque diagnostico ni manejo de la arteritis de Takayasu en la edad pediátrica en nuestro país. El presente tra- bajo resalta que la historia clínica, los exámenes auxiliares enfocados hacia el problema y el manejo multidisciplinario contribuirá al diagnostico preciso, manejo y seguimiento en esta enfermedad de rara presentación, pero de necesaria evaluación en todo paciente con hipertensión renovascular.

\section{REFERENCIAS BIBLIOGRÁFICAS}

1. Al Abrawi S, Fuillet M, David L, Barral X, Cochat P, Cimaz R. Takayasu Arteritis in children. Ped Rheum. 2008;6:1-5. doi:10.1186/1546-0096-6-17.

2. Numano F, Okawara M, Inomata H. Takayasu's arteritis. Lancet. 2000;356:1023-5. PMID:11041416.

3. Ozen S, Ruperto N, Dillon MJ. EULAR/PReS endorsed consensus criteria for the classification of childhood vasculitides. Ann Rheum Dis. 2006 Jul;65(7):936-4. doi: 10.1136/ard.2005.046300.

4. Takayasu M. A case with peculiar changes of the retinal central vessels [en japonés]. Acta Opthalmic Soc Japan. 1908;12:554-5.
5. Numano F, The story of Takayasu arteritis, Rheumatol. 2002;41:103-6. PMID: 11792888

6. De Pablo P, Garcia-Torres R, Uribe N. Kidney involvement in Takayasu arteritis. Clin Exp Rheumatol. 2007 Jan-Feb;25(1 Suppl 44):S10-4. PMID: 17428356.

7. Al Abrawi S, Fuillet M, David L, Barral X, Cochat P, Cimaz R. Takayasu arteritis in children. Ped Rheum. 2008;6:1-5. doi:10.1186/1546-0096-6-17.

8. Pantell RH, Goodman BW. Takayasu's arteritis: the relationship with tuberculosis. Pediatrics. 1981;67:84-8. PMID:6113574.

9. Lupi E. Arteritis de Takayasu. En: Attie F, Zabel $\mathrm{C}$, Buendia $\mathrm{H}$, editores. Cardiologia Pediátrica, diagnóstico y tratamiento, México DF: Editorial Panamericana; 2001:431-43.

10. Arend WP, Michel BA, Bloch DA, Hunder GG, Calabrese LH, Edworthy SM. The American College of Rheumatology 1990 criteria for the classification of Takayasu arteritis. Arthritis Rheum. 1990 Aug;33(8):1129-34. PMID: 1975175.

11. Ishikawa K. Diagnostic approach and proposed criteria for the clinical diagnosis of Takayasu's arteriopathy. J Am Coll Cardiol. 1988;12(4):964-72. PMID: 2901440.

12. Hata A, Noda M, Moriwaki R, Numano F. Angiographic findings of Takayasu arteritis: new classification. Int J Cardiol. 1996 Aug;54 Suppl:S155-63. PMID: 9119518.

13. Karadag O, Aksu K, Sahin A, Zinni FY, Sener $B$, Inanc N. Assessment of latent tuberculosis infection in Takayasu arteritis with tuberculin skin test and Quantiferon-TB Gold test. Rheumatol Int. 2010 Sep;30(11):1483-7. doi: 10.1007/s00296010-1444-Z.

14. Osman M, Aaron S, Noga M, Yacyshy E. Takayasu`Is arteritis progression on anti-TNF biologics: a case series. Clin Rheumatol. 2011;30:7036. doi: 10.1007/s10067-010-1658-1.

15. Ruperto N, Ozen S, Pistorio A, Dolezalova P, Brogan $P$, Maldonado V. EULAR/PRINTO/PRES criteria for Henoch-Schonlein purpura, childhood polyarteritis nodosa, childhood Wegener granulomatosis and childhood Takayasu arteritis: Ankara 2008. Ann Rheum Dis. 2010;69:790-7. doi: 10.1136/ ard.2009.116624.

Artículo recibido el 14 de enero de 2016 y aceptado para publicación el 22 de febrero de 2016.

Proyecto aprobado por la OEAIDE (Oficina Ejecutiva de Apoyo a la Investigación y Docencia Especializada) del Instituto Nacional de Salud del Niño.

\section{Financiado: Recursos propios.}

\section{Correspondencia:}

Franklin Aranda-Paniora.

Dirección: Calle Alcalá $N^{\circ} 105$, Urbanización Mayorazgo.

Teléfono: 3488453

Celular: 994611102

Mail: franklin.aranda@unmsm.edu.pe 